\section{An editor and high-resolution histogram plotter for the CBM/PET microcomputer}

\author{
D. JAMES CAMPBELL \\ University of Wollongong, Wollongong \\ New South Wales, Australia
}

A program that provides an editor and high-resolution plotter has been written to operate on the CBM/PET with versions $2 / 3$ or 4 BASIC. It should run well on all $\mathrm{CBM} / \mathrm{PET}$ machines with 16 or $32 \mathrm{~KB}$ of RAM memory, in conjunction with the various Commodore disk drives and printers. Cassette tape can be used also. The program will run on the C-64 using the PET emulator. Some readers might find parts of the program useful for other computers or want to modify sections for their own needs. The program listing is divided into sections with REM statements that facilitate ease of modification.

The system provides an effective data-handling capability that makes it possible to input, view, edit, store, retrieve, and print out information with ease. Punching information into a computer from the keyboard is subject to human error, and it is desirable to have a checking system in which a data file being typed can be viewed and, if necessary, corrected before saving. This is particularly important with large files, for which editing facilities are essential if repunching of the whole file is to be avoided when a mistake is made at the keyboard.

The program is used frequently in conjunction with an eight-channel data-logging system that uses a KIM-1 single-board microcomputer. Insect-behavior data are transferred from the KIM to the PET (Campbell, 1983) and saved on eight separate files (Campbell \& Forest, Note 1). These can be viewed using the EDITOR.

Operation. The program is menu based, and its operation requires little explanation. After loading and returning RUN, the first prompt is for the name of the sample. This will appear on printouts. The INPUT menu is then presented. The options are: KEYBOARD, TAPE, DISK. A choice is made by returning the enhanced starting letter for the required input option. After the data have been input, the primary menu is presented and the user again makes a choice. The options are: VIEW, EDIT, APPEND, SORT, CONTINUE. The VIEW option allows the user to examine the data that are given on the screen in a specified block. The user is then retumed to the primary menu, and another choice can be made. Each of

The author's mailing address is: Department of Biology, University of Wollongong, P.O. Box 1144,Wollongong, New South Wales 2500, Australia. the remaining primary menu options has its own menu, as follows:

EDIT-CHANGE, DELETE, INSERT, PORTION, BACK; APPEND-KEYBOARD, TAPE, DISK; SORT-WORDSORT, NUMBERSORT, BACK; CONTINUE-SAVE, FINISH, PRINT, GRAPH, BACK.

The BACK option allows the user to move back to the primary menu. This increases the flexibility of the system and avoids the problem of the user's entering the wrong primary menu option and not being able to leave it without performing an unwanted operation. Some options have suboptions. For example, in the SAVE option, it is possible to save to tape or disk.

In saving a file to disk, it might be desirable to write over a preexisting file after editing. If this is done, the following disk error message is given: "FILE EXISTSFIX PROBLEM AND CONT." It is possible in these circumstances to scratch the existing disk file and continue with the save by returning CONTINUE. The updated files is then saved under its original name.

A histogram of the data is obtained by returning $G$ in the CONTINUE menu. After providing details for the axes' names, $x$-axis values, and scaling requirements, the graph can be sent to the screen or printer. If the choice is for the screen, typing 3 starts the graph. For large files, scrolling enables the whole data set to be viewed. Scrolling can be started and stopped by typing 1 , and a retum to the primary menu is achieved by typing 2 while the histogram is scrolling. The spacing between bars on a hardcopy printout can be adjusted by altering the value of CHR $\$()$ on line 2690 of the program. $\mathrm{CHR} \$(36)$ gives standard spacing.

The DIMENSION statement on line 150 of the program is for a sample size of 200 . This can be changed according to requirements. The program needs about $11 \mathrm{~KB}$ of RAM memory for a sample of this size.

The CBM/PET and C-64 microcomputers have a graphics set that is used for generating the high-resolution histogram (line 3330 of the program). This line might cause some trouble for those wishing to type the program from the listing. The graphics symbols that are entered between each set of quotation marks are, consecutively: blank, S\%, S4, S5, S!, RS6RO, RS*RO, RS'RO, where $S$ denotes SHIFT, $R$ denotes RVS, and RO denotes RVS OFF.

Readers with 80-column machines should remove line 1030 from the program; otherwise, the CHANGE option in the EDIT menu will not function correctly when more than 40 columns of a datum line are used.

The BASIC INPUT statement used in the program truncates data after (, or :), and these symbols should be avoided. Care should also be taken when using (").

Availability. A listing of the program is available on request. A cassette tape of the program is available for $\$ 8.00$ to cover costs. Note that the cassette-tape 
format is compatible with any CBM/PET machine and with the C-64 using the PET emulator.

\section{REFERENCE NOTE}

1. Campbell, D. J., \& Forest, J. The KIM-1 microcomputer as an eight channel data logger. Manuscript submitted for publication, 1983.

\section{REFERENCE}

CAMpbell, D. J., Machine language programs for high-speed transfer of data between the KIM-1 and the CBM/PET microcomputers. Behavior Research Methods \& Instrumentation, $1983,15,547-548$.

(Revision accepted for publication November 7, 1983.) 